\title{
Clinical survey of a pedigree with hereditary multiple exostoses and identification of $E X T-2$ gene deletion mutation
}

\author{
WENTAO WANG $^{1 *}$, MINGYUAN YANG $^{1 *}$, YUHANG SHEN $^{1 *}$, KAI CHEN $^{1 *}$, \\ DONGHUA WU ${ }^{1}$, CHANGWEI YANG ${ }^{1}$, JINYI BAI ${ }^{1}$, DAWEI HE ${ }^{1}$ and JUN GAO $^{2}$
}

Departments of ${ }^{1}$ Orthopedics and ${ }^{2}$ Digestion, Shanghai Changhai Hospital, Shanghai 200433, P.R. China

Received January 6, 2022; Accepted February 10, 2022

DOI: $10.3892 / \mathrm{mmr} .2022 .12657$

\begin{abstract}
The aim of the present study was to report a clinical survey of hereditary multiple exostoses (HME) in a large Chinese pedigree, and the identification of a novel deletion mutation of exostosin glycosyltransferase 2 (EXT-2) gene. A patient with multiple exostoses with huge cartilage-capped tumors in scapula, knees and ankles received surgery in Department of Orthopedics (Shanghai Changhai Hospital). A total of 20 family members were recruited to the study, with seven members (five male; two female) diagnosed as HME. The family members of the patients with HME were examined, clinical data and peripheral blood samples were collected, and their DNA was sequenced. The incidence of HME in this family pedigree was $35 \%$. Exostoses were most frequently in the tibiae with occurrence in six patients, followed by ribs, femurs, radii, fibulae, scapulae and humeri. DNA sequencing of peripheral blood revealed a novel deletion mutation, c.824-826delGCA, in exon 5 of the EXT-2 gene, which was observed in all the patients with HME, but not in the healthy family members. Several characteristics of HME in the pedigree were observed, such as susceptibility of male gender, decreased average age of onset and height and increased severity of clinical symptoms with generations.
\end{abstract}

\section{Introduction}

Hereditary multiple exostoses (HME) is an autosomal dominant skeletal disorder that is characterized by multiple

Correspondence to: Professor Dawei He, Department of Orthopedics, Shanghai Changhai Hospital, 168 Changhai Road, Yangpu, Shanghai 200433, P.R. China

E-mail: hedawei2000@sina.com

Professor Jun Gao, Department of Digestion, Shanghai Changhai Hospital, 168 Changhai Road, Yangpu, Shanghai 200433, P.R. China E-mail: 13816012151@163.com

*Contributed equally

Key words: hereditary multiple exostoses, pathogenesis, clinical survey, DNA sequencing cartilage-capped tumors growing outward from the metaphyseal region of the long tubular bones and other skeletal structures $(1,2)$. HME can lead to physical and psychological problems in patients, causing huge burdens to both families and society, although it is a rare disease $(1,3,4)$. The incidence of HME was reported to be $0.4-1$ per 50,000 in the Western countries $(1,5)$.

The appearance of a palpable mass near the knees, shoulders, ankles or wrists is the most common clinical presentation in patients with HME (1). Hennekam (6) suggest that a patient with HME is generally burdened with an average of six exostoses. In addition to patient appearance D'Ambrosi et al (3) reported that children affected by HME report lower sports activity, particularly among female patients. In addition, neurological syndromes, pains, functional disorders and deformities are also common clinical presentations of HME (7-9). According to previous studies, although most of the masses were benign, $\leq 2 \%$ of cases develop into malignant chondrosarcoma or osteosarcoma $(1,10)$. These data and results were summarized and reported in western countries; however, few reports of clinical features of HME have been studied in Chinese populations. Therefore, it is essential to study the clinical course of this disease in Chinese populations and compare the similarities and differences in natural history, clinical presentation and characteristics with other ethnicities to facilitate a further understanding of HME. This is one of the objectives of the present study.

Study of pathogenesis is useful for further understanding of diseases and provides guidance for treatments. As to the pathogenesis of HME, a number of risk factors have been identified and pathogenic mechanisms have been speculated (10-15), such as genes and genetic factors, abnormal embryonic development, and growth and development disfunction of epiphysis at long bones. For example, Inubushi et al (16) reported a connection between increased BMP signaling and osteochondromagenesis, which serves an essential role in skeletal development by regulating chondrocyte proliferation and differentiation; they also suggest that treatment with a BMP inhibitor may be effective in patients with HME, which showed promising results in their study conducted on mouse models. Bukowska-Olech et al (12) hypothesized that heparanase, an enzyme that cleaves the heparan sulfate (HS) chains and stimulates chondrogenesis, is physiologically found only in the hypertrophic zone and perichondrium, and its wider 
distribution and increased activity possibly served a role in the development of osteochondromas.

Although several risk factors and pathogenic mechanisms have been hypothesized, it is most frequently suggested that mutations in exostosin glycosyltransferase 1 (EXT-1) and $E X T$-2, genes with autosomal dominant inheritance, are the most likely responsible factors for $\operatorname{HME}(2,12)$, having been detected in $28-65 \%$ and in $21-61 \%$ of the affected patients, respectively (1). EXT-1 is located on chromosome 8q24,11-q24.13 and EXT-2 on chromosome 11p11-12 (11); both encode glycosyltransferases and serve vital roles in the synthesis of HS. HS is a polysaccharide material that binds to core proteins to produce HS proteoglycans, which are present to a large extent in the cell membrane and extracellular matrix and interact with BMPs participating in the regulation of bone and cartilage formation (11). Therefore, heterozygous mutations of the EXT-1 or EXT-2 gene lead to an HS deficiency of $\sim 50 \%$, which is inadequate for giving rise to osteochondroma formation in patients with HME (11). An increasing number of mutations of EXT-1 and EXT-2 have been observed $(12,17)$. In Guo et al (18), a novel heterozygous splice mutation (c.1284+2del) in EXT-1 was identified in a three-generation family with HME. Medek et al (10) identified different EXT-1 gene mutations in five probands that resulted in premature stop-codons (p.Gly124Argfs*65, p.Leu191*, p.Trp364Lysfs*11, p.Val371Glyfs*10, p.Leu490Profs*31). In two of the probands, nonsense mutations were found in EXT-2 gene (p.Val187Profs*115, p.Cys319fs*46). In total, five mutations were novel and two mutations have occurred de novo in probands. Xian et al (19) also found a novel heterozygous splice site mutation $(c .1173+2 \mathrm{~T}>\mathrm{A})$ in the EXT-2 gene. Chen et al (20) identified an original nonsense mutation in the EXT-2 gene (9c.526C $>$ T; p.Gln176*) in a Chinese family with HME from Hangzhou. Indeed, $>70 \%$ of HME cases arise from monoallelic mutations in either EXT-1 or EXT-2 $(10,11,21)$, and in $5-34 \%$ of the patients with HME, mutations EXT-1 or $E X T-2$ are not detected (22), suggesting that there might be other mutations of EXT genes in patients with HME which may serve important roles in pathogenesis.

Therefore, the object of the present study was to report the clinical presentations and characteristics of HME in a large Chinese pedigree and to analyze the molecular background using DNA sequencing in this pedigree.

\section{Materials and methods}

Patients and a large Chinese pedigree. The proband was a 24-year-old female patient from Anhui, Jiangsu (China), with multiple exostoses involving the scapula, bilateral distal femurs and bilateral proximal tibiae and bilateral proximal fibula, who received osteochondroma surgery for the scapula at Shanghai Changhai Hospital (Shanghai, China) in 2012 and osteochondroma surgery for the bilateral proximal tibiae and fibula in 2016 due to pain, functional limitation and deformity. Post-biopsy analysis indicated that the masses were multiple exostoses. Several generations of family members from the proband were investigated by two surgeons (WW and YS). A total of 20 family members were recruited in the present study, including 10 males and 10 females (age, 6-83 years). The dates patients were recruited started at 9 March 2017 and ended at
28 March 2017. Diagnostic criterium was X-ray examination revealing that the long bone metaphysis had at least two or more osteochondromas. In addition, three patients (two males and one female, age, 45 to 55 years old) with arthritis and without HME who received surgery in Shanghai Changhai Hospital in June-December 2017 were recruited and their cartilage specimens were resected and used as controls. The present study was approved by the Institutional Review Board of Shanghai Changhai Hospital (approval no. CHEC20180184), and all patients provided written informed consent for the study. In addition, since minor patients were not able to understand the study design, purpose and cautions of this study, the parents of the patients provided informed consent.

Collection of data, post-operation biopsy and peripheral blood. The authors (WW and YS) went to Jiangsu (China) to collect data on 9 March 2017 and 28 March 2017, respectively. Each participant in this pedigree underwent careful physical and/or radiographic examinations by two surgeons (MY and $\mathrm{DH})$. Multiple exostoses were diagnosed as at least two osteochondromas, arising from the lateral ends of the humerus, ulna, femur, tibia, fibula, knee joint or scapula. Those patients with multiple exostoses were classified using a the multiple osteochondromas classification system through a Switching Neural Networks approach (23). Clinical data were collected from this family pedigree, including the number of patients with multiple exostoses and healthy subjects, age, height, the age when the first exostosis was recognized, deformity or functional disabled and locations of exostoses.

Resection surgery was performed in the scapula and bilateral knees of the proband (III-9) and multiple exostoses were resected in bilateral knees of the patient III- 8 for pains and deformity. These surgeries were performed by the same surgeon $(\mathrm{DH})$ and the postoperative biopsy was conducted by the Department of Pathology at Shanghai Changhai Hospital. These two patients were followed up at two years after the operation.

In addition, EDTA-anticoagulated peripheral blood $(5 \mathrm{ml})$ was drawn from each subject of this pedigree for DNA sequencing.

Genetic analysis. Preliminary whole-genome sequencing in three HME patients (II-3, III-8 and III-9) showed that the deletion mutation at c.824-826delGCA in the EXT-2 gene (in exon 5 of the EXT-2) was the only mutation site in these three HME patients by comparing with the published EXT-1 and EXT-2 reference sequences from Leiden Open Variation Database (LOVD; databases.lovd.nl/shared/genes), which might have disastrous effects on the etiology of HME. According to preliminary results (data not shown) the deletion mutation at c.824-826delGCA in the EXT-2 gene may affect production of glycosyltransferases, which are vital for the synthesis of heparin sulfate, and interaction with bone morphogenetic protein that participates in the regulation of bone and cartilage formation. However, the exact mechanism by which this deletion mutation influences the occurrence and development of HME needs further study. Therefore, to explore whether this mutation at c.824-826delGCA, in exon 5 of the EXT-2 was the only mutation in this pedigree, genomic DNA was extracted from peripheral blood leukocytes using 


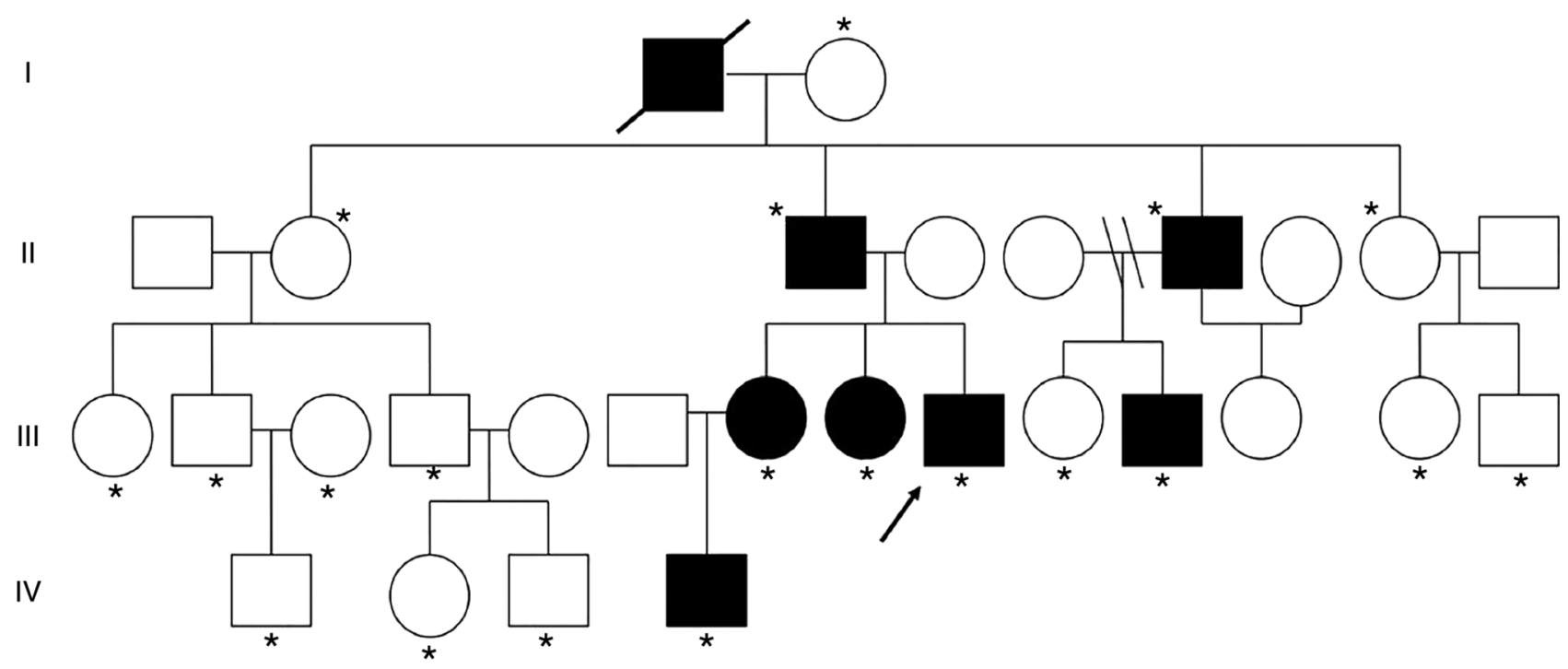

Figure 1. Pedigree of the family with HME. The proband (III-9) is indicated with an arrow. Boxes or circles with an oblique line and double lines indicate that the individual is deceased and divorced, respectively. $\mathbf{m}$, affected male; $\bullet$, affected female; $\square$, healthy male; $\circ$, healthy female. "Family members included in this study.
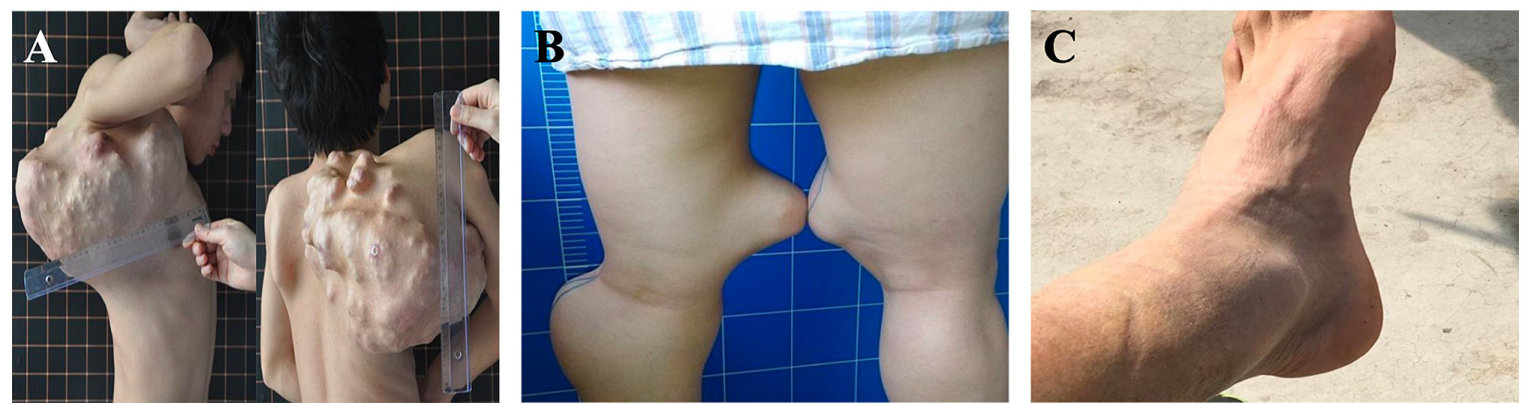

Figure 2. Clinical pictures of three HME patients (II-3, III-8 and III-9). (A) Posterior and lateral film of a large chondrosarcoma in right scapula in proband (III-9), which was diagnosed as a malignant cartilage-capped tumor by post-operation biopsy. (B) Clinical pictures of chondrosarcomas in bilateral distal femurs and left proximal tibia in proband's sister (III-8). (C) Clinical pictures of chondrosarcoma in left distal tibia in proband's father (II-3).

the GeneJET Whole Blood Mini kit (Thermo Fisher Scientific, Inc.) according to the manufacturer's protocol. The exon 5 of the EXT-2 gene including the mutation site was amplified by PCR using the upstream primer 5'-CTGGGAAGTAAGGAA AGGG-3' and the downstream primer 5'-GAGGTGGGAAAG ATGGGTG-3' (amplicon length: 497; annealing temperature: $\left.54^{\circ} \mathrm{C}\right)$. The amplifications were performed to a final volume of $20 \mu \mathrm{l}$, containing $1 \mu \mathrm{l}$ genomic DNA, $10 \mu \mathrm{l} 2 \mathrm{X}$ Premix Taq (Vazyme Biotech Co., Ltd.), $1 \mu \mathrm{l}$ of each primer and $7 \mu \mathrm{l}$ sterilized distilled water. The thermocycling conditions were as follows: Initial denaturation of $5 \mathrm{~min}$ at $95^{\circ} \mathrm{C}$; followed by 35 cycles of $30 \mathrm{sec}$ at $95^{\circ} \mathrm{C}, 45 \mathrm{sec}$ at annealing temperature and $30 \mathrm{sec}$ at $72^{\circ} \mathrm{C}$; and a final extension for $10 \mathrm{~min}$ at $72^{\circ} \mathrm{C}$. Direct sequencing of the PCR products was performed using BigDye Terminator v3.1 (Applied Biosystems) with the ABI $3730 \mathrm{XL}$ automated sequencer (Applied Biosystems).

\section{Results}

Clinical characteristics of the pedigree. A total of 20 family members were recruited in the present study with seven members (five male and two female) diagnosed as HME with the incidence of 35\%; these include II-3, II-6, III-7, III-8, III-9, III-11 and IV-4 (Fig. 1). The grandfather (I-1) of the proband also had HME according to medical records; however, he died in 2016 so was not recruited. The pedigree of this family with HME was shown in Fig. 1. The proband (III-9) was a 24-year-old male patient with multiple exostoses involving the scapula, bilateral distal femurs, and bilateral proximal tibiae and fibula (Fig. 2) and other family members was subject without HME.

In this pedigree, the number of male patients with HME was larger than that of female HME patients. All the patients with HME in second generation were males and all the male descendants of II generation with HME (II-3 and II-6), named as III-9 and III-11 were diagnosed as HME. These findings suggested that males were more susceptible to HME compared with females.

The age of all patients when the first exostosis was recognized was $<10$ years old (Table I). The average age of second generation, third generation and fourth generation when the first exostosis was recognized was 8.5 years old, 5.25 years old and 4 years old, respectively, indicating that the age of onset of HME became earlier in each generation. 


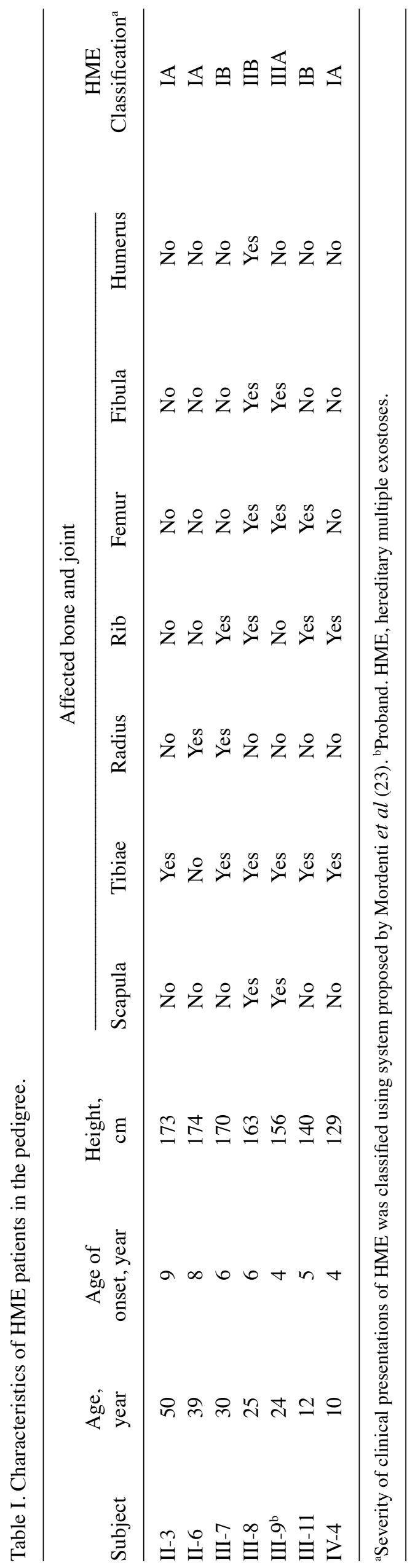

The height of II-3, II-6, III-7, III-8 and III-9 was 173, 174, 170, 163 and $156 \mathrm{~cm}$, respectively (Table I). Patients III-11 and IV-4 were juveniles; based on the data of other five adult patients with HME, it was found that the height of each affected subject might have a decreasing trend with generations. Whether the height of patients III-11 and IV-4 will be shorter than that of second generation needs further follow-up.

In this pedigree, exostoses were most frequently in the tibia with occurrence in six patients $(85.7 \%)$, followed by rib (4/7; 57.1\%), femur (3/7; $42.9 \%)$, radius $(2 / 7 ; 28.6 \%)$, fibula (2/7; $28.6 \%)$ and scapula $(2 / 7 ; 28.6 \%$; Table I). Exostoses was also found in humerus in III-8.

Subjects were classified according to Switching Neural Networks approach for classification (23) (Table I). The results showed that the clinical presentation of each of the four patients in the third generation was classified as IB, IIB, IIIA and IB, which was more serious compared with that of second generation, both IA. Taken together, the data suggest that the younger patients exhibited more severe conditions compared with the others. The clinical presentation of III-9 (proband, 24 years old) and III-8 (26 years old) was classified as IIIA and IIB, respectively, which was more severe than that of the older relatives, III-7, II-6 and II-3. The clinical presentation of proband was the most serious because she had multiple exostoses involving the scapula, bilateral distal femurs and bilateral proximal tibiae and fibula.

Biopsy and two years' follow-up. Patients III-8 and the III-9 (proband) received resection surgery in Shanghai Changhai Hospital for the pains and skeletal deformations caused by multiple exostoses in the scapula and the bilateral proximal tibiae and fibula of the proband, and in the bilateral proximal tibiae and fibula of the patient III- 8 . The masses resected from the scapula and bilateral proximal tibiae and fibula of the proband were verified as malignant cartilage-capped tumor and benign exostoses, respectively. The post-operation biopsy of the bilateral proximal tibiae and fibula in patient III-8 indicated that the resected masses were benign exostoses. The intraoperative pictures and biopsy are shown in Fig. 3A and B. As shown in Fig. 3A, patient III-9 (proband) was placed in lateral position, and huge masses could be seen in his scapula. The masses from proband's scapula were resected, and the postoperative biopsy showed that it was malignant cartilage-capped tumor. The tumor cells were arranged in small clusters with occasional binuclear nuclei, some lobular margins contained tumor cells with obvious heteromorphism, with nuclear hypertrophy, deep staining, occasional multinucleated and binucleated giant cells. Cortical destruction and surrounding soft tissue infiltration were seen. As shown in Fig. 3B, patient III-8 suffered from huge masses in the bilateral proximal tibiae and fibula, and the masses from the bilateral proximal tibiae and fibula were resected and the postoperative biopsy showed that the tumor tissue was lobulated and composed of chondrocytes and cartilage matrix. At the 2-year follow-up, no recurrence of multiple exostoses was observed in either patient with the significant improvements of quality of life (no pain and improved activity ability) in these patients according to the follow-ups of these patients, as shown in Fig. 3C.

Genetic analysis of EXT-2. A 3-bp heterozygous deletion (GCA) at the 267-269 of EXT-2 was found in HME patients 

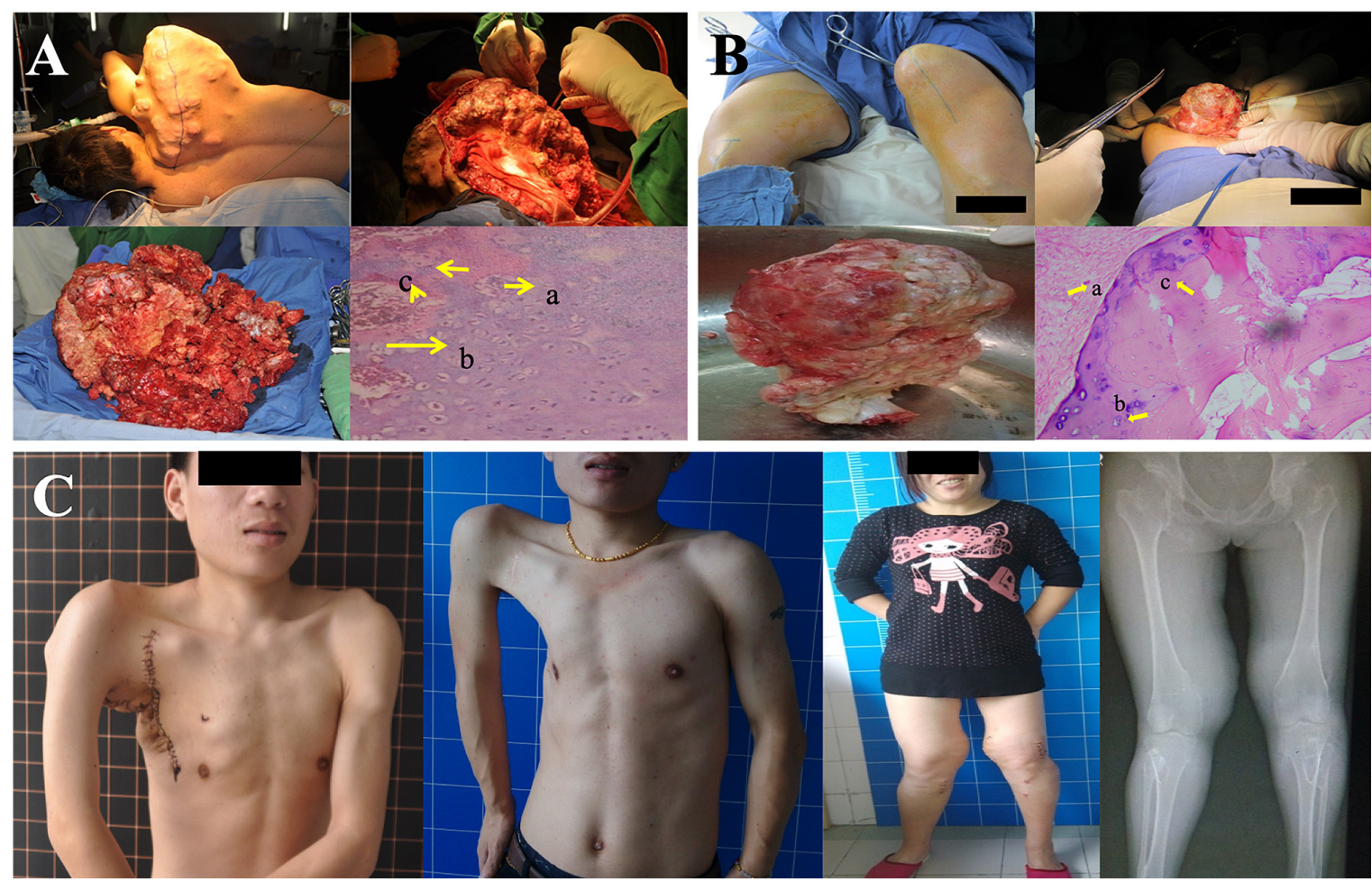

Figure 3. Intraoperation pictures, postoperative biopsy and clinical pictures at two-years' follow-up in three HME patients (II-3, III-8 and III-9). (A) Intraoperation pictures of the proband (III-9). The masses from proband's scapula were resected, and the postoperative biopsy showed that it was malignant cartilage-capped tumor. The tumor cells were arranged in small clusters with occasional binuclear nuclei, some lobular margins contained tumor cells with obvious heteromorphism, with nuclear hypertrophy, deep staining, occasional (a) multinucleated and (b) binucleated giant cells. Cortical destruction and surrounding soft tissue infiltration were seen (c). (B) The intraoperation pictures of the patient III-8. The masses from the bilateral proximal tibiae and fibula were resected and the postoperative biopsy showed that the tumor tissue was lobulated (a) and composed of chondrocytes (b) and cartilage matrix (c). (C) At the two-years follow-up, no recurrence of multiple exostoses was observed in the proband (left) or in patient III-8 (right).

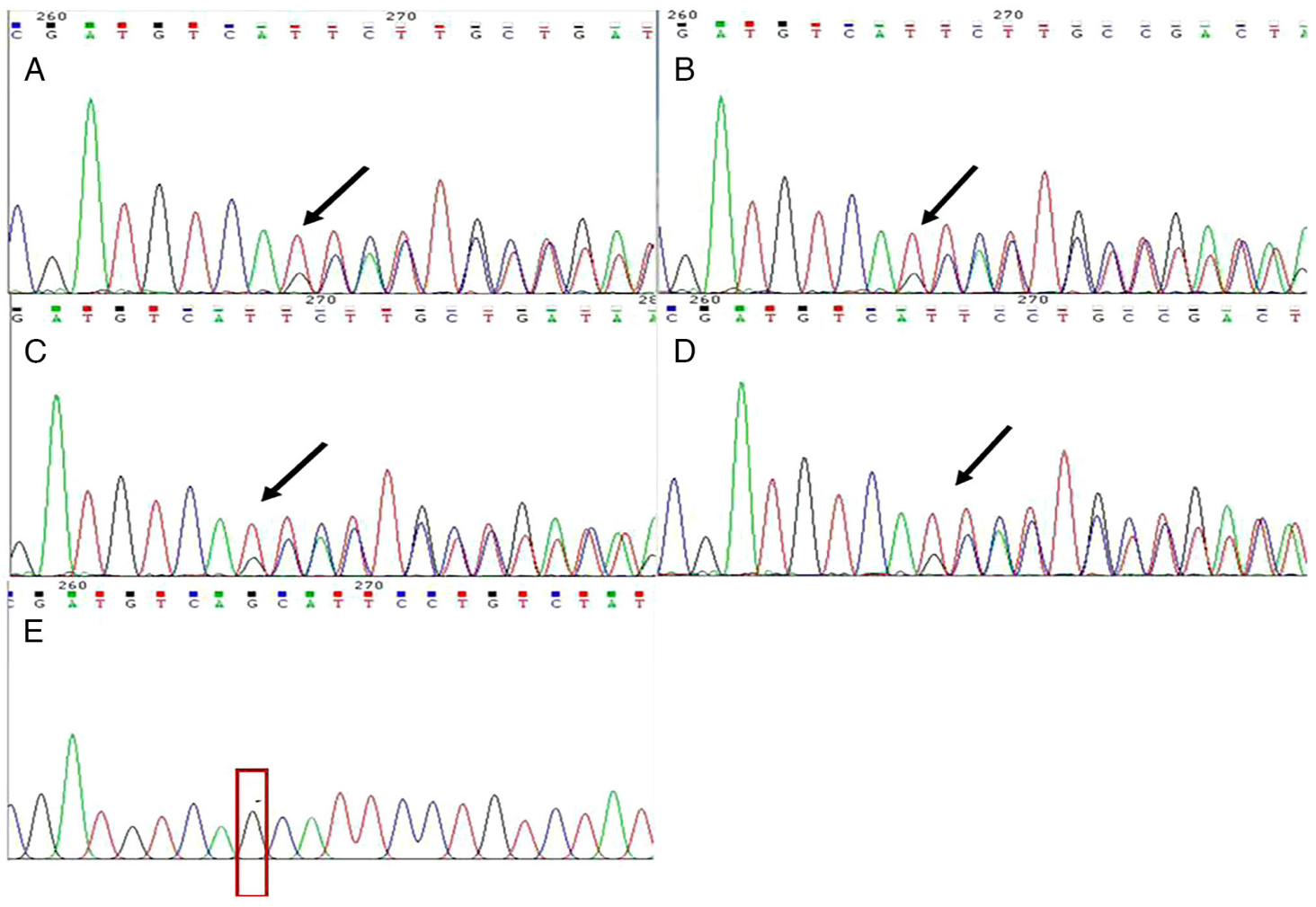

Figure 4. Sanger DNA sequencing results of patients (A) II-6, (B) IV-4, (C) III-11, (D) III-7 and (E) unaffected subject (II-8). In parts A-D, a 3-bp heterozygous deletion (GCA) at the 267-269 of EXT-2 was found. Beyond the point of nucleotide 267, two overlapping sequences were observed: ATGTCAGCATTCCT and ATGTCATTCCT, indicating that the deletion was a heterozygous event; however, no overlapping sequences was observed in the unaffected subject (II-8, red box). Arrows indicate the beginning of overlapping sequences in patients with HME. 
(Fig. 4A-D). Beyond nucleotide 267, two overlapping sequences were observed: ATGTCAGCATTCCT and ATGTCATTCCT, indicating that the deletion was a heterozygous event; however, no overlapping sequences was observed in the unaffected subject (II-8, Fig. 4E). After genetic analysis of c.824-826delGCA at EXT-2 in all family members in this pedigree except for III-8, III-9 and II-3 who received Whole-Genome Sequencing, The results showed that deletion mutation at c.824-826delGCA in the exon 5 of EXT-2 gene was detected in four of the affected individuals (II-6, III-7, III-11 and IV-4), whereas none of the unaffected family members examined carried this mutation (I-2, II-2, II-8, III-1, III-2, III-3, III-4, III-10, III-13, III-14, IV-1, IV-2 and IV-3). Thus, this finding was consistent with preliminary results (III-8, III-9 and II-3). This mutation was found in all patients (II-3, II-6, III-7, III-8, III-9, III-11 and IV-4) rather than in unaffected family members, suggesting that the mutation site at c.824-826delGCA in the EXT-2 gene was the causative mutation for HME in this family. No other mutations were reported in any of the analyzed samples.

\section{Discussion}

The present study surveyed 20 family members of four generations in a large pedigree with seven subjects diagnosed with HME and the relatives without HME used as controls. The study not only reported clinical characteristics of HME, providing valuable baseline assessments of the nature and characteristics of HME, but also found a mutation of EXT-2 gene in a large Chinese pedigree, extending the known mutational spectrum of EXT-2 gene for improved understanding of the genetic basis of patients with HME.

Compared with other studies in which unrelated healthy subjects, matched for geographical ancestry, were included as controls $(21,24)$, the present study recruited family members without HME, which decreased the selection bias and the results were more reliable. Moreover, seven out of the twenty family members examined were found to have multiple exostoses with the incidence of $35 \%$ in this pedigree. The incidence of HME in total populations was reported to be $0.4-1$ per 50,000 in Western populations and 40 out of 50,000 in the Chamorro people (1). Guo et al (25) investigated the epidemiology of HME in mainland China by searching databases. In their study, a total of 1,051 cases with more detailed documentations were reported in mainland China since 1990, among which $4 \%$ were diagnosed as sporadic vs. 96\% as familial cases and the estimated penetrance was 39\%. Whatever the incidence in pedigree or in total populations, it varies in different studies since these data were taken from hospital reports and health databases of the affected patients and families, with a focus on people who reported the symptoms and were classified as affected. Therefore, studies with large sample size in multicenter and various ethnicities are necessary.

A total of five out of seven (71.4\%) patients with HME in this pedigree were males, indicating that males might be more susceptible to HME compared with females, which was consistent with previous studies $(1,21,24,26)$. Guo et al (25) performed a systematic review by searching several databases and the prevalence was higher in males (male:female ratio 637:414; 1.5:1), which might provide more reliable information for the susceptibility of HME in different sexes. However, these findings were proved wrong by other authors $(27,28)$. For example, Wicklund et al (27) showed that the male to female ratio was unskewed in nuclear families (probands, affected sibs, and parents). The present study recommended that males should be paid more attention by physical and/or radiographic examinations, especially in HME pedigrees, for early diagnosis and treatment.

The median age of patients with HME in this pedigree was 26 years-old, with the oldest age at 50 years-old (II-3) and youngest age of 10 years-old (IV-4). Xia et al (21) reported a median age of 31, with the oldest and youngest in their pedigree at 62 and 25 years old, respectively. In Ruan et al (24), the youngest patient was 6 years old and the oldest was 75 years old. This difference between the present study and theirs might be due to the time point of the survey and the health problems of the subjects. It is hypothesized that, in general, most patients are diagnosed before the age of 12 and the median age of diagnosis is 3 years old $(1,29)$. Guo et al (25) also indicates that most of patients with HME (83\%) were diagnosed by the age of $\leq 10$ years (six cases detected at birth) and $14 \%$ of patients were diagnosed by the age of 10-20 years. The results of the present study showed that the age of onset varied from 4-9 years-old, which was consistent with the general consensus, including Guo et al (25). According to these findings, early diagnosis and early treatment should be paid attentions in hereditary susceptible subjects.

The present study also reported the height of patients with HME and the results of adult affected patients suggested that the height of each adult subject might have a decreased trend in subsequent generations, consistent with Clement et al (30) and Ruan et al (24). Since few studies have examined the relationship between patients' height throughout generations, it is hypothesized that the present study might provide valuable baseline assessments of the nature and characteristics of HME.

Patients are often diagnosed with HME by a physician following the appearance of a palpable mass near the joints where is the lateral side of the most active growth plate of a long bone (31). Knees, shoulders, ankles and wrists are the most commonly affected joints $(1,25,26)$. The present study found that tibiae, femurs, radii and fibulae were the primary locations of exostoses. These findings suggested that if patients come to clinics with masses in these locations and with a family history, HME should be considered. However, ribs are also a common location of exostoses in this study, which was inconsistent with Guo et al (25). That study showed that ribs were less often involved with the incidence of $17 \%$ in studied populations. Selection of patients might be the primary contributor. In Beltrami et al (31), osteochondromas were often first discovered on the ribs and the proximal tibia, where they can be clearly visible and palpable, which might be an another reason. In addition, other locations including pelvis, vertebrae and carpal/tarsal bones were reported in previous studies $(26,32)$, whereas these locations were not observed in the pedigree of the present study. Recently, surgeons have also reported osteochondroma at rare locations, such as $\mathrm{C} 1$ and $\mathrm{C} 2(33,34)$. It has been reported that a patient with HME is generally burdened with an average of six exostoses (1), and the results of the present study were consistent with this finding. Facial bones are largely unaffected, which may be due to intramembranous 
ossification at these locations (35), and no exostoses were found in these bones in the present study. All these findings indicated that clinical characteristics of HME often varied among individuals, and exostoses could occur at several bones and joints, which could be a great challenge for surgeons.

Although HME may be asymptomatic, a wide spectrum of severity of clinical manifestations are found in patients with this disorder $(31,36)$. The present study showed that the severity of the disease varied in pedigree by using the classification system of Mordenti et al (23). In the present study, the severity of clinical presentations in patients with HME was associated with the generation, as it was found that the patients whose conditions were more severe were third or fourth generation. The results were consistent with Shen et al (26), who hypothesized that the patients whose conditions were more severe were younger than the others. Therefore, the younger patients with HME in third or fourth generations should be paid more attention since their clinical symptoms might be more severe and treatments should be performed earlier.

Malignant transformation was only found in one case, the proband (III-9), though it is relatively rare. Beltrami et al (31) showed that the places of malignant transformation more frequently involved were the pelvis, the scapula, the proximal part of the femur and the humerus. In addition, the authors of that study hypothesized that malignant transformation in HME is diagnosed at a younger age than chondrosarcomas are in patients without HME, most often in the early 20 s, which was also verified in the present study in the 24-year-old proband with chondrosarcomas in the scapula. In addition, no recurrence of chondrosarcomas and exostoses was observed at the two-years follow-up in the proband and in the patient III-8, indicating that surgical resection may be a useful method for exostoses and may greatly affect the quality of life of patients with HME; this was consistent with Liang et al (32).

The present study performed Sanger sequencing) on 20 family members in a large HME pedigree to screen for potential gene mutations and to expand our knowledge of the mutation hotspots of EXT-2; for example, c.824-826delGCA, in exon 5 of the EXT-2, according to the findings of preliminary experiments (data not shown). The present study showed that the c.824-826delGCA EXT-2 deletion mutation was found in all patients with HME in this pedigree, but not in the unaffected family members, and was also the only mutation site in the patients with HME. This mutation site of EXT-2 was first found and reported by us, indicating that EXT-2 may serve a key important role in the pathogenesis of HME, which were consistent with Xia et al (21) who found a novel heterozygous frameshift mutation, c.119_120delCT (p.Thr40ArgfsX15), in exon 2 of the EXT2 gene in HME patients with HME and hypothesized that EXT-2 mutation might be responsible for more cases of HME in the Chinese population. In addition, it is hypothesized $(1,2)$ that mutations of either EXT-1 or EXT-2 will critically disrupt the HS chain elongation process and subsequently the function of numerous proteins, such as fibroblast growth factor, vascular endothelial growth factor and transforming growth factor- $\beta(1,2)$. These aberrant proteins include a number of important signaling receptors and paracrine growth, proliferation and differentiation factors involved in various normal and pathological processes such as morphogenesis, angiogenesis, inflammation and tumor metastasis. Therefore, the findings of the present study may extend the EXT-2 mutational spectrum in HME pathogenesis. However, how this novel deletion mutation of EXT-2 influences the pathogenesis of HME and what signaling pathways requires further study.

The severity of HME varies, even within the same family whose affected members all share the same mutation (36). The present study was consistent with Porter et al (36), as c.824-826delGCA of EXT-2 gene was observed in all patients with HME however severe their clinical symptoms. One previous study (37) reported that the malignant transformation of preexisting osteochondromas relied on wild-type cells with functional EXT; that is, secondary peripheral chondrosarcomas occur through EXT-independent mechanisms. This may be the reason that the exostoses in the scapula of the proband (III-9) was a malignant cartilage-capped tumor, which is a severe complication of HME, whereas other family members in this pedigree exhibited less severe symptoms.

Although some new findings have been explored in the present study, the sample size was relatively small and further studies at a larger scale are required.

In conclusion, the present study reported clinical characteristics of patients with HME in a large Chinese pedigree and identified a novel c.824-826delGCA of EXT-2 gene in this family. Results from the present study increased our understanding of the nature of HME, extended the known mutational spectrum of EXT-2 and suggested further application of mutation screening in genetic counseling and subsequent prenatal diagnosis of HME.

\section{Acknowledgements}

Not applicable.

\section{Funding}

The present study was supported by The National Natural Science Foundation of China (grant nos. 81572636 and $82102525)$.

\section{Availability of data and materials}

All data generated or analyzed during the present study are included in this published article.

\section{Authors' contributions}

WW, MY, YS and KC performed the screening and data collection procedure. DW and JB performed statistical analysis. WW, MY and CY performed data interpretation and drafted the manuscript. WW, MY, YS, KC, DW, JB, DW, JG and CY confirm the authenticity of all the raw data. DH and JG designed the study and supervised the performance of the study. DH and JG confirm the authenticity of all the raw data. All authors have read and approved the final manuscript.

\section{Ethics approval and consent to participate}

The present study was approved by the Institutional Review Board of Shanghai Changhai hospital (approval number CHEC20180184). 


\section{Patient consent for publication}

All patients or their parents/guardians in the present study provided written informed consent for the study and publication of patient information and images.

\section{Competing interests}

The authors declare that they have no competing interests.

\section{References}

1. D'Arienzo A, Andreani L, Sacchetti F, Colangeli S and Capanna R: Hereditary multiple exostoses: Current insights. Orthop Res Rev 11: 199-211, 2019.

2. Komura S, Matsumoto K, Hirakawa A and Akiyama H: Natural history and characteristics of hand exostoses in multiple hereditary exostoses. J Hand Surg Am 46: 815.e1-815.e12, 2021.

3. D'Ambrosi R, Caldarini C, Ragone V and Facchini RM: Effect of multiple hereditary exostoses on sports activity in children. J Orthop 15: 927-930, 2018.

4. D'Ambrosi R, Ragone V, Caldarini C, Serra N, Usuelli FG and Facchini RM: The impact of hereditary multiple exostoses on quality of life, satisfaction, global health status, and pain. Arch Orthop Trauma Surg 137: 209-215, 2017.

5. Ryckx A, Somers JF and Allaert L: Hereditary multiple exostosis. Acta Orthop Belg 79: 597-607, 2013.

6. Hennekam RC: Hereditary multiple exostoses. J Med Genet 28: 262-266, 1991.

7. Clement ND and Porter DE: Can deformity of the knee and longitudinal growth of the leg be predicted in patients with hereditary multiple exostoses? A cross-sectional study. Knee 21: 299-303, 2014.

8. Noonan KJ, Feinberg JR, Levenda A, Snead J and Wurtz LD: Natural history of multiple hereditary osteochondromatosis of the lower extremity and ankle. J Pediatr Orthop 22: 120-124, 2002.

9. Matsumoto Y, Matsumoto K, Harimaya K, Okada S, Doi T and Iwamoto Y: Scoliosis in patients with multiple hereditary exostoses. Eur Spine J 24: 1568-1573, 2015.

10. Medek K, Zeman J, Honzík T, Hansíková H, Švecová Š Beránková K, Kučerová Vidrová V, Kuklík M, Chomiak J and Tesařová M: Hereditary multiple exostoses: Clinical, molecular and radiologic survey in 9 families. Prague Med Rep 118: 87-94, 2017.

11. Tepelenis K, Papathanakos G, Kitsouli A, Troupis T, Barbouti A, Vlachos K, Kanavaros P and Kitsoulis P: Osteochondromas: An updated review of epidemiology, pathogenesis, clinical presentation, radiological features and treatment options. In Vivo 35: 681-691, 2021.

12. Bukowska-Olech E, Trzebiatowska W, Czech W, Drzymała O, Frąk P, Klarowski F, Kłusek P, Szwajkowska A and Jamsheer A: Hereditary multiple exostoses-a review of the molecular background, diagnostics, and potential therapeutic strategies. Front Genet 12: 759129, 2021

13. Sinha S, Mundy C, Bechtold T, Sgariglia F, Ibrahim MM, Billings PC, Carroll K, Koyama E, Jones KB and Pacifici M: Unsuspected osteochondroma-like outgrowths in the cranial base of hereditary multiple exostoses patients and modeling and treatment with a BMP antagonist in mice. PLoS Genet 13: e1006742, 2017.

14. Piombo V, Jochmann K, Hoffmann D, Wuelling $M$ and Vortkamp A: Signaling systems affecting the severity of multiple osteochondromas. Bone 111: 71-81, 2018.

15. Mundy C, Chung J, Koyama E, Bunting S, Mahimkar R and Pacifici M: Osteochondroma formation is independent of heparanase expression as revealed in a mouse model of hereditary multiple exostoses. J Orthop Res: Jan 7, 2022 (Epub ahead of print).

16. Inubushi T, Lemire I, Irie F and Yamaguchi Y: Palovarotene inhibits osteochondroma formation in a mouse model of multiple hereditary exostoses. J Bone Miner Res 33: 658-666, 2018.
17. Al-Zayed Z, Al-Rijjal RA, Al-Ghofaili L, BinEssa HA, Pant R, Alrabiah A, Al-Hussainan T, Zou M, Meyer BF and Shi Y: Mutation spectrum of EXT1 and EXT2 in the Saudi patients with hereditary multiple exostoses. Orphanet J Rare Dis 16: 100, 2021.

18. Guo X, Lin M, Yan W, Chen W and Hong G: A novel splice mutation induces exon skipping of the EXT1 gene in patients with hereditary multiple exostoses. Int J Oncol 54: 859-868, 2019.

19. Xian C, Zhu M, Nong T, Li Y, Xie X, Li X, Li J, Li J, Wu J, Shi $\mathrm{W}$, et al: A novel mutation in ext 2 caused hereditary multiple exostoses through reducing the synthesis of heparan sulfate. Genet Mol Biol 44: e20200334, 2021.

20. Chen Z, Ruan W, Li M, Cao L, Lu J, Zhong F and Bi Q: A novel nonsense mutation in the EXT2 gene identified in a family with hereditary multiple osteochondromas. Genet Test Mol Biomarkers 24: 478-483, 2020.

21. Xia P, Xu H, Shi Q and Li D: Identification of a novel frameshift mutation of the EXT2 gene in a family with multiple osteochondroma. Oncol Lett 11: 105-110, 2016.

22. Ishimaru D, Gotoh M, Takayama S, Kosaki R, Matsumoto Y, Narimatsu H, Sato T, Kimata K, Akiyama H, Shimizu K and Matsumoto K: Large-scale mutational analysis in the EXT1 and EXT2 genes for Japanese patients with multiple osteochondromas. BMC Genet 17: 52, 2016.

23. Mordenti M, Ferrari E, Pedrini E, Fabbri N, Campanacci L, Muselli M and Sangiorgi L: Validation of a new multiple osteochondromas classification through switching neural networks. Am J Med Genet A 161A: 556-560, 2013.

24. Ruan W, Cao L, Chen Z, Kong M and Bi Q: Novel exostosin-2 mutation identified in a Chinese family with hereditary multiple osteochondroma. Oncol Lett 15: 4383-4389, 2018.

25. Guo XL, Deng Y and Liu HG: Clinical characteristics of hereditary multiple exostoses: A retrospective study of mainland chinese cases in recent 23 years. J Huazhong Univ Sci Technolog Med Sci 34: 42-50, 2014.

26. Shen Y, Zhang L, Chen B, Dong L, Wang Y and Wang S: Novel deletion and $2397 \mathrm{G}>\mathrm{T}$ mutations of the EXT1 gene identified in two Chinese pedigrees with hereditary multiple exostoses using exon sequencing. Transl Pediatr 9: 619-628, 2020.

27. Wicklund CL, Pauli RM, Johnston D and Hecht JT: Natural history study of hereditary multiple exostoses. Am J Med Genet 55: 43-46, 1995.

28. Kumar A, Jain VK, Bharadwaj M and Arya RK: Ollier disease: Pathogenesis, diagnosis, and management. Orthopedics 38: e497-e506, 2015.

29. Schmale GA, Conrad EU III and Raskind WH: The natural history of hereditary multiple exostoses. J Bone Joint Surg Am 76: 986-992, 1994.

30. Clement ND, Duckworth AD, Baker AD and Porter DE: Skeletal growth patterns in hereditary multiple exostoses: A natural history. J Pediatr Orthop B 21: 150-154, 2012.

31. Beltrami G, Ristori G, Scoccianti G, Tamburini A and Capanna R: Hereditary multiple exostoses: A review of clinical appearance and metabolic pattern. Clin Cases Miner Bone Metab 13: 110-118, 2016.

32. Liang C, Wang YJ, Wei YX, Dong Y and Zhang ZC: Identification of novel EXT mutations in patients with hereditary multiple exostoses using whole-exome sequencing. Orthop Surg 12: 990-996, 2020.

33. Tbini M,Lahiani R, Kharrat O, Riahi I and Bensalah M: Anterior C1 osteochondroma. Joint Bone Spine 89: 105305, 2021.

34. Jemel N, Gader G, Bedioui A, Zammel I and Badri M: C1 C2 spinal cord compression in hereditary multiple exostoses: Case report and review of the literature. Int J Surg Case Rep 89: 106576, 2021.

35. Stieber JR and Dormans JP: Manifestations of hereditary multiple exostoses. J Am Acad Orthop Surg 13: 110-120, 2005.

36. Porter DE, Lonie L, Fraser M, Dobson-Stone C, Porter JR, Monaco AP and Simpson AH: Severity of disease and risk of malignant change in hereditary multiple exostoses. A genotype-phenotype study. J Bone Joint Surg Br 86: 1041-1046, 2004.

37. de Andrea CE, Reijnders CM,Kroon HM, de Jong D, Hogendoorn PC Szuhai K and Bovée JV: Secondary peripheral chondrosarcoma evolving from osteochondroma as a result of outgrowth of cells with functional EXT. Oncogene 31: 1095-1104, 2012.

This work is licensed under a Creative Commons Attribution-NonCommercial-NoDerivatives 4.0 International (CC BY-NC-ND 4.0) License. 